\title{
THE IMPACT OF VIEWING IMAGES OF PRECEDENTS ON THE COGNITIVE PROCESS OF ARCHITECTURAL IDEA GENERATION
}

\author{
Djari, Chahinez (1,2); Arrouf, Abdelmalek (1,2) \\ 1: Laboratory of architectural and urban phenomena modeling and studying (LEMPAU); 2: University \\ El Hadj Lakhder, Batna1, Batna, Algeria.
}

\begin{abstract}
Among the increasing number of researches about design thinking, several studies, empirically investigate the report between design process and different sources of inspiration. Visualization of Images represents one of the most current stimuli in the architectural design

This work focuses on the link between the active part of design process and images of precedents when visualized by the designer at the beginning of his design activity. It aims to identify and measure the impact of such visualization on the cognitive process of ideation.

We use the protocol analysis method. Data are collected through design experiment and coded by the semio-morphic coding scheme. Results show that the visualization of images of precedents enhances the productivity of the ideation process. The process consistency is also improved by the apearence of homogeneous phases. Moreover the ideation process becomes more creative cognitively, by making the genesis of primitive chains of actions faster, easier and similar.

Accordingly, this paper communicate the effect of a common practice such images' visualization on the architectural design process to get insight on the cognitive befits of this practice.
\end{abstract}

Keywords: Design process, Design cognition, Visualisation, Idea generation, Creativity

\section{Contact:}

Djari, Chahinez

University of Batna 1

architecture and urbanism

Algeria

c.ninez@hotmail.com

Cite this article: Djari, C., Arrouf, A. (2019) 'The Impact of Viewing Images of Precedents on the Cognitive Process of Architectural Idea Generation', in Proceedings of the 22nd International Conference on Engineering Design (ICED19), Delft, The Netherlands, 5-8 August 2019. DOI:10.1017/dsi.2019.24 


\section{INTRODUCTION}

In the practice of architectural design, perceived information differs from the design project data (land, program, planning regulations...) to architectural precedents already produced by other designers in different design situations. The design process depends on both the nature of the perceived information, and their time of introduction in the design process.

This paper aims to understand the relationship between the idea generation and the information perceived at the beginning of the design work. The major research issue raised in this paper wonder if images visualization of architectural precedents derived from internet, upstream design work, has or has not an impact on the cognitive content of architectural idea generation process.

We are interested to explore the initialization phase of the design process through its cognitive content to understand the effect of the image visualisation on the design cognition content. We consider design activity as a semio- morphic production process, which takes the form of a complex system of interrelated cognitive operations (Arrouf, 2006, 2012). We use cognitive measurement to present the impact of the images visualization of architectural precedents upstream of the design work on the idea generation process.

\section{RELATED WORKS}

Images considered as a source of inspiration were the subject of several studies to identify their impact on the produced designs. Cai Hui and al (2010) classified their impact is into two groups. The first considers the sources of inspiration as design aids. The second group considers the sources of inspiration as a cause of fixation. In fact, the majority of these works measure the impact of stimuli on design outcome mainly.

In another hand, the studies that focus on the cognitive content of design process: "aims to reveal what the designer sees, does and think at the moment of conception (Goldschmidt (1991), Schön and Wiggins (1992), Suwa, Purcell and Gero (1998)). It "focuses on the study of the cognitive and informational content of the design activity"' (Arrouf, 2006, 2012).

The particularity of our study is the combination of the previous two approaches. We aim to understand the effect of the image visualisation on the design cognition content. More accurately, we measure the impact of images visualization of architectural precedents derived from internet, upstream design work, on the cognitive process of architectural idea generation, instead of the design outcome itself.

In this research, we consider that the idea generation enclose all the cognitive process of formation and elaboration of architectural design. We use new parameters to evaluate productivity, homogeneity and creativity of the cognitive design content.

\section{PROTOCOL ANALYSIS}

This approach belongs to design science field introduced by Herbert Simon 1969, which study the cognitive process of the design thinking. The original version of protocol analysis incite the participant to verbalize his thoughts aloud, simultaneously to his process of problem solving (Mc Neill, Gero and Warren, 1998). "This method has been adopted by several works: Eastman (1970), Cross (1984), Ullman et al. (1988), Eckersley (1988), Goldschmidt (1991), Cross et al. (1992), McGinnis and Ullman (1992), Schön and Wiggins (1992), Van Someren et al. (1994), Cross et al. (1996) Suwa and Tversky (1996, 1997), Suwa, Gero and Purcell (1998, 1999, 2001, 2002), Dorst et al. (2001) and Tang et al. (2002)" (Arrouf, 2006).

Jiang et al (2009), shows the growing number of publications that have used the method of protocol analysis, since its emergence until 2009. In other hand, he mentions the validation of the method by "Delphi workshop" as a crucial turning point, which raised it as the most used empirical method in design research.

The adaptation of this method originally developed to study cognitive activity of problem solving to the characteristics of the design activity by different researchers, generated different approaches (Hay et al, 2017). The process-oriented approach focuses on the description of the design process. As for to the content-oriented approach interested in the content of the design process (Arrouf, 2006, 2012).

This work adopts the content-oriented approach and focuses on the cognitive modification of design process actions, after image visualization of precedents. 
From studies about the design process, it results various models developed by Mc Neill, Gero, and Warren (1998), Suwa, Purcell and Gero (1998) and the model that we are using is the one developed by $\operatorname{Arrouf}(2006,2012)$.

\subsection{Coding scheme}

The coding scheme based on eleven informational categories that allows a rich and detailed codification of the cognitive content of the designing process. Arrouf (2012) define those categories as below:

1. External Perception Category (PE):

It belongs to the cognitive level of perception. "It saves design actions information's that uses the perception mechanisms" dealing with the project data.

2. $\quad$ Prior Knowledge Reference Category (RE):

This action category highlights the use of information derived from prior learning of the designer "The cognitive sciences have indicated that any cognitive task undertaken by human beings is conducted through previous knowledge from the domain to which the task belongs (...) two main classes of actions are identified: the procedural reference actions and the substantive reference actions" (Arrouf, 2012).

3. Abstract Design action Category (CA):

It belongs to the semantic level and it contains six types of actions that govern the production of meaning through objectives, choices, strategies, decisions and pertinence.

4. Sense Production Action Category (CAS):

It belongs also belongs to the semantic cognitive level; it gives and produce meaning during the design work. "It brings together conceptual actions that make sense in the design activity. It records the design actions that deal with non-morphic information (abstract notions)" (Arrouf, 2012).

5. Sense figuration category (FS):

It contains a single design action that produces meaning figures. It is not related to the shape of the object in design. "It groups, meaning figuration actions that produce either abstract figures that are commonly called ambiguous figures, or figures that schematics. It is something other than the shape of the object in design, such as diagrams, charts or tables" (Arrouf, 2012).

6. Internal perception Category (PI):

It belongs to the cognitive level of perception, but unlike the category of external perception, it is concerned with the perception that takes place within the design process. It contains two subclasses; the first is non-visual and concerns "the actions that allow designer to establish connections between his own virtual representations". The second subclass is visual and concerns the perception of the figurative representations produced by the designer. It "carries actions of perception with a visual foundation and enable the functioning of visual thinking" (Arrouf, 2012).

7. Internal Knowledge Reference Category (RI):

It allows the designer to create references and to use them by "constituting a reservoir of knowledge specific to the particular design situation and actions. That permit the exploitation of his reservoir, which corresponds to "short-term memory"' (Arrouf, 2012).

8. Internal Knowledge Reuse Action Category (RCI):

This category contains a single design action that allows the designer to "go back, over the time of the process, to reuse information or knowledge previously generated" (Arrouf, 2012). This category, unlike the internal perception, permits going back further in time of the process.

9. Morphic Design Action Category (CM):

This category belongs to the gathering of the morphic, the semantic and the perceptive levels. It contains the same abstract design actions that are now "applied to the morphic conformation of the object in design"

10. Morphic Figuration Category (FM):

This category concerns the actions of object figuration. It contains four types of actions that correspond to different graphic productions, plus translation action, that allows the passage between the meaning and its figuration.

11. Evaluation Actions Category (CR):

This category evaluates the various morphic productions, compared to the design process aims, strategies, and pertinences previously produced. 


\section{EXPERIENCE}

The experimental work consisted on holding a design session for a given subject (Suwa and Tversky, 1997, Suwa, Gero and Purcell, 1998., Tang and Gero, 2002). The participants to this experience were six volunteer architects', three men and three women with an experience of two years each. The experiment involved the execution by two groups of architects, of two separate and different design works.

For the first group it took the form of a regular order for designing a bus shelter from the usual data specific to these situations. While for the second group, in addition to the data presented by the design task, the designers receive images of bus shelters previously derived from the internet and grouped together in a PowerPoint ${ }^{\circledR}$ file.

This choice is due to the fact that the web is becoming the most obvious option to get inspired (Herring et al, 2009) and it provides visual alternative that is easily accessible, richer and faster than a traditional research on revue or periodic. The designers visualizes these images at will, for a maximum of ten minutes, before starting the design work.
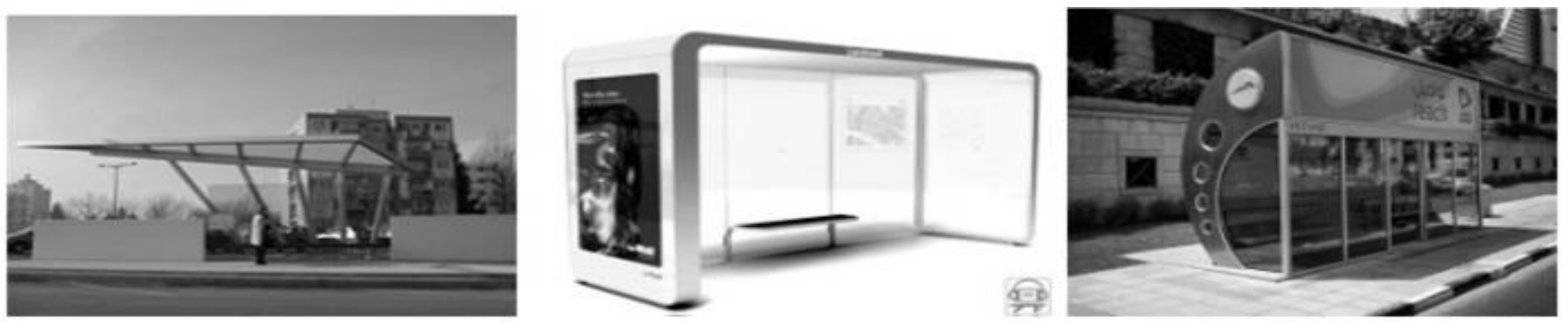

Figure 1. Examples of bus shelters images derived from the internet, provided to the second group of designers.

\subsection{Procedure}

We drove an experiment accordingly to the guidelines provided by McNeill, Gero and Warren (1998). Solely one protocol among the three collected is here analysed. We used the double-blind randomized method in order to ensure the objectivity of this choice. The two selected processes are (SV1) the process without image visualization and (AV2) with image visualization.

\subsection{Analysis of the protocols}

The description of collected data according to the standard method of protocol analysis consists of two major moments: segmentation and codification (Arrouf, 2006; 2012).

\subsubsection{Segmentation and codification}

Among segmentation techniques, this work achieves an intention-oriented segmentation, used by: Goldschmidt, (1991); Purcell and al (1994); Suwa and Tversky, (1997); Purcell and Gero, (1998); Mc Neill, Gero and Warren, (1998); Arrouf (2006). In this approach, "any change in the designer's intentions suggested by the content of his thoughts or by his action marks the beginning of a new segment" (Arrouf, 2006).

Conformably to Arrouf coding scheme, we code the cognitive content of each segment according to its correspondent action category, as shown in Table 1. The double codification of protocols eliminate the relativity of the operation. However, for reasons of availability of coders and feasibility over time, we choose the two successive codifications methods. In addition, to consensus both codifications, an arbitration is realized to obtain the final codification of protocols (McNeill, Gero \& Warren, 1998).

Table 1. Description model (segment 2 extract from SV1 process codification).

\begin{tabular}{|c|c|c|c|c|c|}
\hline \multirow[t]{2}{*}{$\mathrm{N}^{\circ}$} & \multirow[t]{2}{*}{ time } & \multicolumn{2}{|c|}{ Segmentation } & \multicolumn{2}{|r|}{ Codification } \\
\hline & & $\begin{array}{l}\text { Verbalisation and } \\
\text { action }\end{array}$ & Graphic productions & $\begin{array}{l}\text { Action } \\
\text { category }\end{array}$ & Justification \\
\hline 2 & $00: 34$ & $\begin{array}{l}\text { A bus shelter (she } \\
\text { writes a bus shelter) }\end{array}$ & & PI & $\begin{array}{l}\text { Non-visual } \\
\text { perception of the } \\
\text { previous segment }\end{array}$ \\
\hline
\end{tabular}




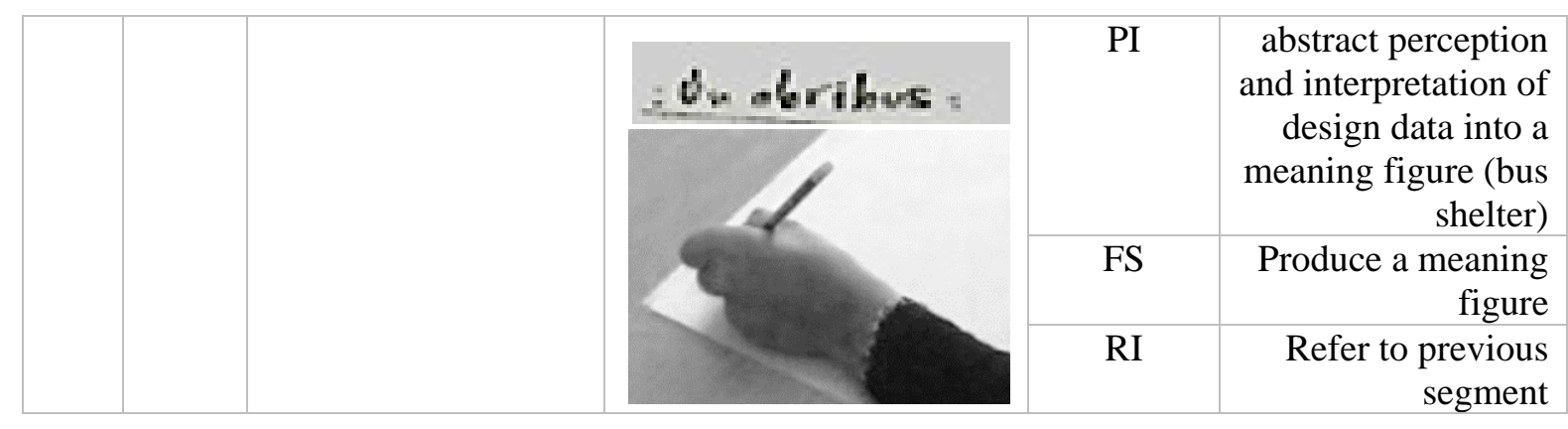

\subsubsection{Data handling}

First, we used the "actions chain" defined by Arrouf (2006) to represent segments of the design process, by the action categories occurrence. It consisted of eleven binary values that reflect the cognitive solicitation of the eleven action categories. The ' 0 ' indicated the absence of the category, while the ' 1 ' indicated its presence in the relevant segment. Thus, the chain of actions of segment 1 , was taken as an example: 10000000000 and the one of segment 3 is: 01111110000 according to Table 2.

Table 2. the occurrence of the actions categories excerpt from (SV1) protocol.

\begin{tabular}{|l|c|c|c|c|c|c|c|c|c|c|r|}
\hline Segments & PE & RE & CA & CAS & FS & RI & PI & RCI & FM & CM & CR \\
\hline 1 & 1 & 0 & 0 & 0 & 0 & 0 & 0 & 0 & 0 & 0 & 0 \\
\hline 2 & 0 & 0 & 0 & 0 & 1 & 0 & 1 & 0 & 0 & 0 & 0 \\
\hline 3 & 0 & 1 & 1 & 1 & 1 & 1 & 1 & 0 & 0 & 0 & 0 \\
\hline
\end{tabular}

Second, we also used the "primitive chains" defined by Arrouf $(2006,2012)$ as the "basic chains" of each process. They were the chains that remain after the elimination of repeating actions chains. The set of these primitive action chains was the "deep structure of the process" (Arrouf, 2006, 2012) while the set of action chains of all segments was called "the surface structure of the process".

\section{RESULTS}

The comparison between the two selected processes: (SV1) without image visualization and (AV2) with image visualization, takes place in three steps. The first measure the influence degree of images visualization of precedent designs on the cognitive productivity of the process. It uses the measurements of efficiency and effectiveness, recommended by Goldschmidt (1995) as measures of productivity. The second step compares the cognitive aspect of the two designs processes. When the last step compares the cognitive creativity through primitive chains of Arrouf $(2006,2012)$.

\subsection{Productivity of the design process}

Goldschmidt (1995) accept Pritchard and Watson's notion that is to measure productivity "the important issues to look at are efficiency and effectiveness". She also indicates that efficiency correspond to the economy of invested design actions and saving the path length to achieve the desired creative result. This is why we measure the efficiency of the cognitive process at two levels.

In the first level, we compare the distances of the design "paths" driven during each activity in order to achieve a result. We use the number of segments in each design process, to measure the length of cognitive path.

Figure 2 shows that viewing images of precedents matches with design process length saving.

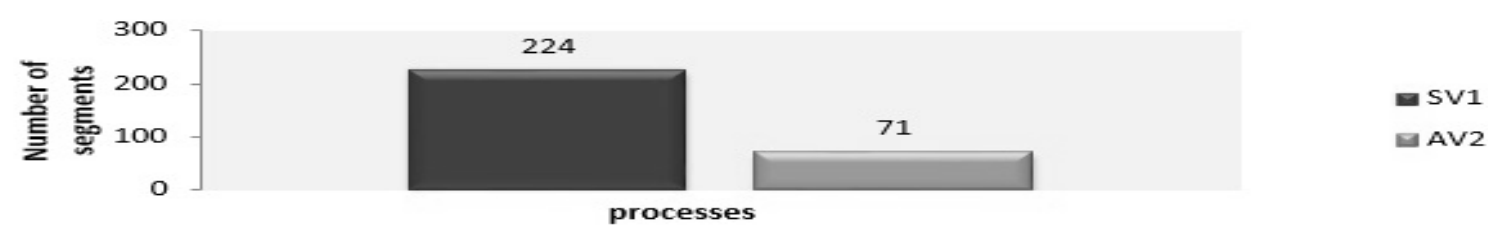

Figure 2. Number of segments per process, for the two analysed process SV1 and AV2. 
In the second level of measuring efficiency, we compare between the cognitive content of the twodesign process SV1 an AV2.

Figure 3, represents the average occurrence of the eleven actions categories of the semio-morphic coding scheme, in each design process. The main differences are:

1. The averages occurrence of the external reference category (RE), which contains the reference actions to designer prior knowledge, show that SV1 makes more reference to external process data than AV2. Thus, it reveals the hesitation of the designer and the inefficiency of knowledge produced by his process.

2. The abstract design category (CA) and the sense figuration category (FS) are more solicited by SV1 than by AV2. This means that the choices, decisions, strategies and relevance identified by SV1 do not have the same operational potential as in AV2, where few abstract actions lead to work that is more morphic.

3. The hesitation of SV1 reappears by the high occurrence percentage of Internal Knowledge Reuse (RCI) category. It indicate more distant and frequent backtracking, compared to the process time length.

4. The high occurrence rate of the evaluation actions category (CR) also shows the lack of assurance of the process that needs to make repeated assessments to ensure the quality of its solutions.

5. The morphic activity, visible through the occurrence of the two categories: $(\mathrm{CM})$ morphic design and (FM) morphic representation is more intense within AV2 process than within SV1 process. However, the morphic activity even in AV2 taken apart is more important than the semic activity. In SV1 process, the semic and morphique activities are almost equivalent with a slight preponderance for the semic activity.

This means that visualizing images of precedents makes the process more efficient cognitively. It reduces the semic production, which becomes operative, less hesitant and more decisive. On the other hand, it increases the morphic activity that absorbs most of the activity of the process.

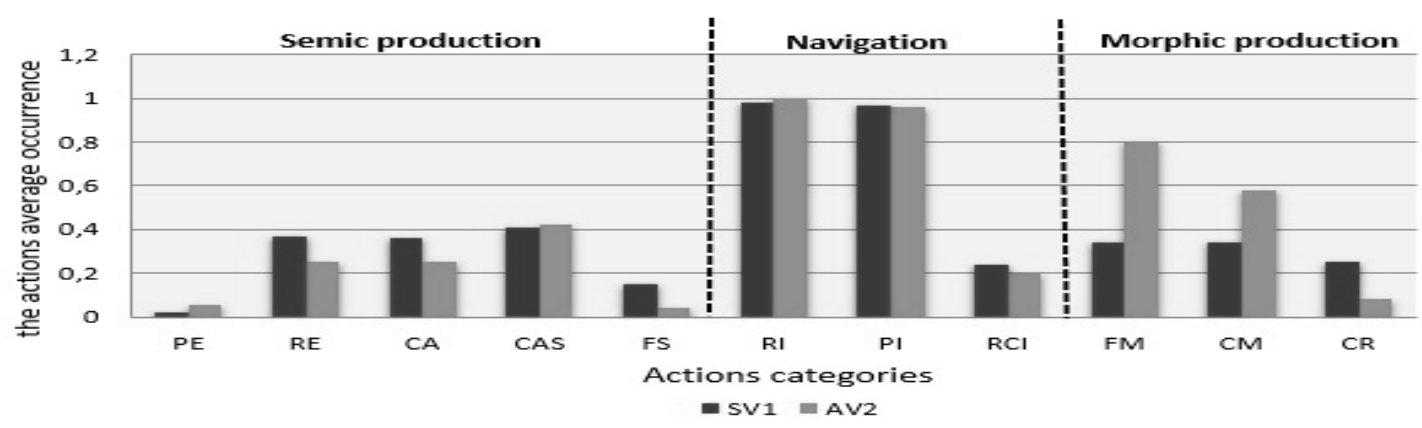

Figure 3. The average occurrence of each action categories for SV1 and AV2 processes.

The contraction of the process length is due to an economy in the number of invested design actions, especially semic ones, which become more effective.

Consequently, viewing images of precedents suggest a tendency of increasing the efficiency of the process, enriching the semic experience of the designer, reinforcing his morphic work and making the studied process more mature.

Figure 4 shows that visualization reduces the hesitation of the designer by lengthening the duration of his process segments. This corresponds to an increase of the efficiency of the designing process which is equal to more thoughtful process with a uniform, less hesitant and more operative rhythm.

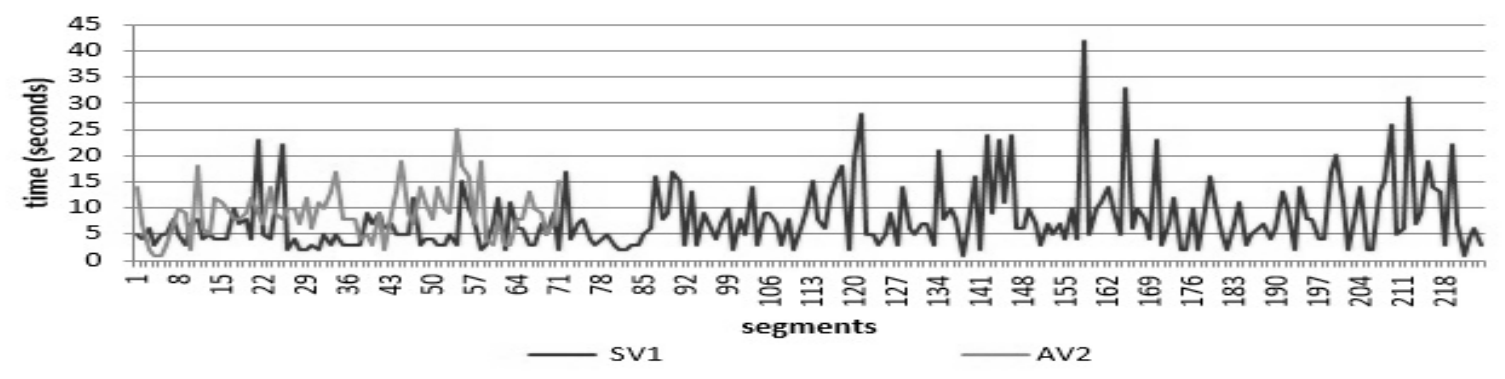

Figure 4. The duration of the segments of the two collections (SV1) and (AV2). 
The notion of effectiveness is vaguely explain by Goldschmidt (1995), This is why we use the synonym "infallibility" to develop a mean to measure effectiveness. Infallible "says something that cannot fail to produce the expected result" (Lexis, 1994). It is the capacity for an action to achieve the desired result without the need to redo or repeat it. In this work, we measure effectiveness by comparing the number of repetitions of the different categories of actions during the two types of designing processes.

Figure 5 shows the average occurrence percentage of each actions' category in the primitive chains and in the rest of repeated chains in SV1 and AV2 design processes.

Total elimination of (PE) and (FS) in the repeated chains of AV2 process, indicates the effectiveness of its semic production. Concerning all the navigation actions categories, there is also a reduction of the percentage of repeated chains in AV2 process. Which indicates an increase of the effectiveness of navigation.

The percentage of the morphic production decreases in the repeated chains in AV2 process, mainly the evaluation category (CR), implies a high effectiveness of both evaluation action as well as morphic production. The simultaneous decrease of the repetition percentage in all the actions categories of AV2 implies an increase of the process effectiveness.

The images visualization of precedents makes the process more effective by increasing the effectiveness of its semic production, its navigation and its morphic production.

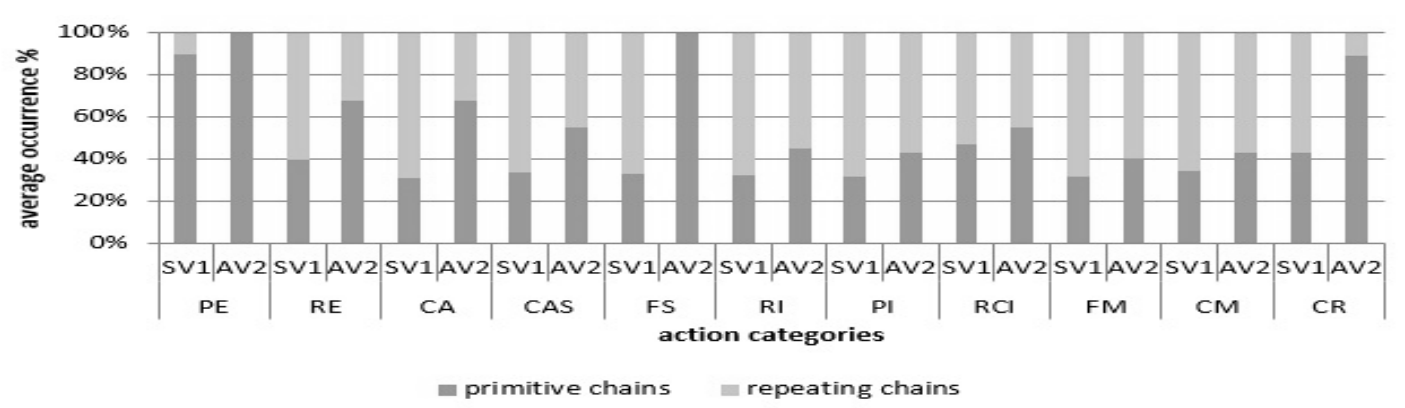

Figure 5. The average occurrence percentage of the eleven actions categories according to the semio-morphic coding scheme, in both primitive chains and repeated chains of SV1 and AV2 design processes.

Overall, the efficiency and effectiveness makes the studied process more productive.

\subsection{The morphological aspect of design process}

Figure 6 and Figure7 represents' the surface graph of the cognitive content of AV2 and SV1 design processes'. The distribution of the eleven actions categories shows the cognitive morpholoy of the design process, according to their chronological order of appearance.

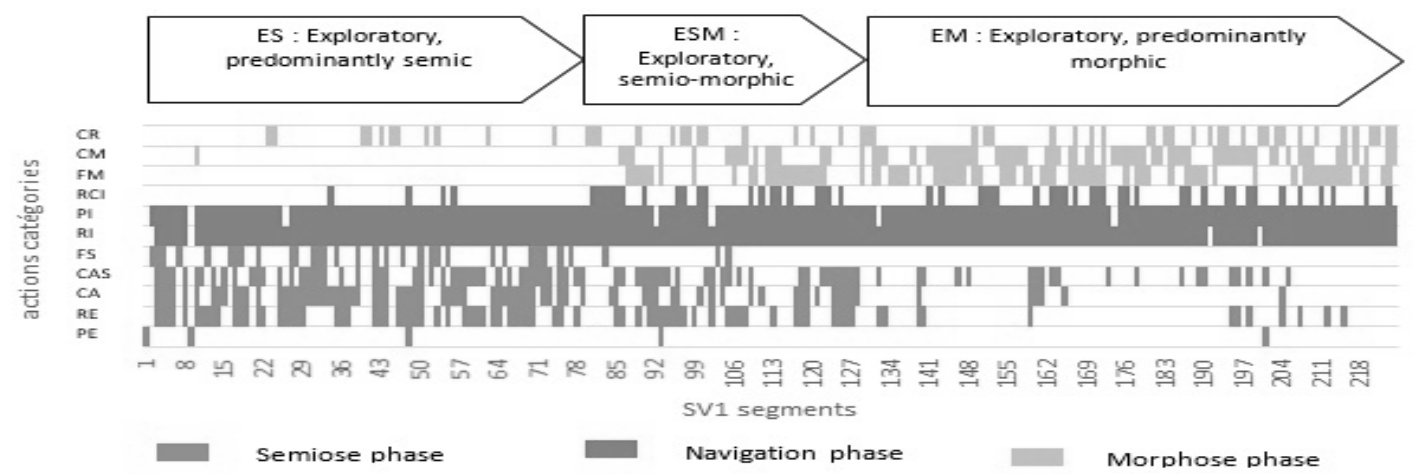

Figure 6. The morphology of the SV1 process, illustrated by the homogeneous and exploratory phases.

Both design processes presents' a similar morphology with an analogous distribution of the actions category. Which manifests by a strong solicitation of the PE, RE, CA, CAS and FS categories at the beginning of both processes and fades as we move forward. Unlike the categories FM, CM, CR that 
exists only after a certain moment and continues until the end. The categories PI and RI persists along the two processes.

Arrouf (2012) has defined two types of phases that allows' to read different moments' of the design process. The first type is "exploratory phase" which solicits a large number of action category and take a non-homogeneous form. The second type is "homogeneous phase", it is more uniform than the first and limited to a single type of production, whether it is semic or morphic.

Despite the global surface similarity of both design processes. We note that SV1 process contains essentially "exploratory phases", that solicit irregular and various categories. We see this in particular in the heterogeneity of its surface aspect in Figure 6, the "homogeneous phases" of SV1 are not long enough to be distinguish .This leads to long exploratory phases that change only dominance.

In the surface graph of AV2 process in Figure 7, the "homogeneous phases" alternate with "exploratory phases". This interchange of the two types of phases in the process AV2 breaks the exploratory sequence that characterizes SV1 process, and attributes more homogeneity to the morphology of AV2.

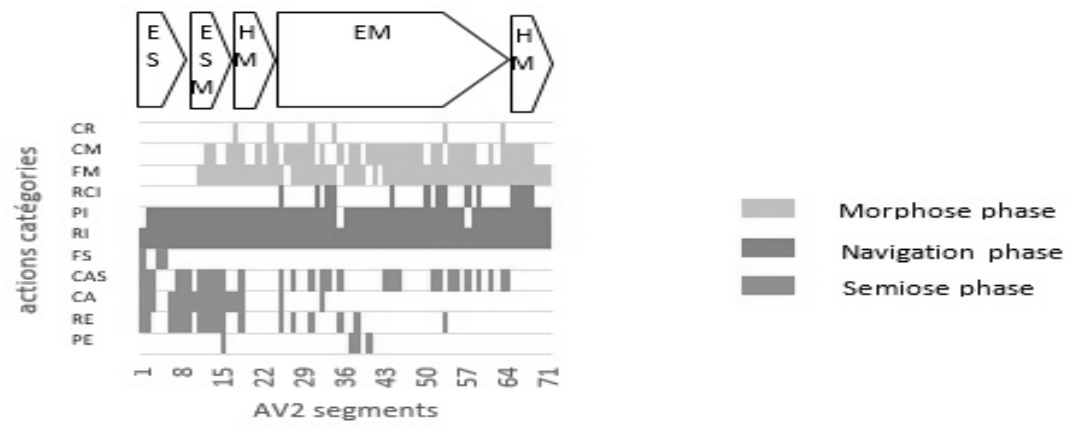

Figure 7. The morphology of the AV2 process, illustrated by the homogeneous and exploratory phases (ES: Exploratory predominantly semic, EMS: Exploratory semiomorphic, HM: homogeneous morphic phase, EM: Exploratory predominantly morphic).

The images visualization of precedents affects the morphology of the studied process by interrupting its exploratory phases, and introducing homogeneity into its cognitive content. It modifies the solicitation order of all action categories, generates a precocity and concatenation of morphic production that dominates the process.

\subsection{The cognitive creativity of the design process}

Most studies on creativity in the field of architectural design consider it as the quality of produced design (Finke, 1990, Casakin \& Goldschmidt, 2000, Goldshmidt \& Smolkov, 2006, Perttula, 2007). In another hand, creativity is also measured as a quality of the design process itself (Goldschmidt, 2005, Arrouf, 2012, Sun et al, 2014).

In this research, we measure the cognitive creativity of the process based on the "primitive chains" previously defined. A process is more creative when it contains more chains that are primitives.

\subsubsection{The creativity degree of the process}

We measure the creativity degree of the process according to the primitive chains percentage in SV1 and AV2. Figure 8 indicates that SV1 primitive chains percentage is less than AV2.

The images visualization of precedents go with the increase of the primitive chains ratio as well reduction of the repetition of the same chains. It increases the creativity degree of the studied process by increasing the ratio of its original chains.

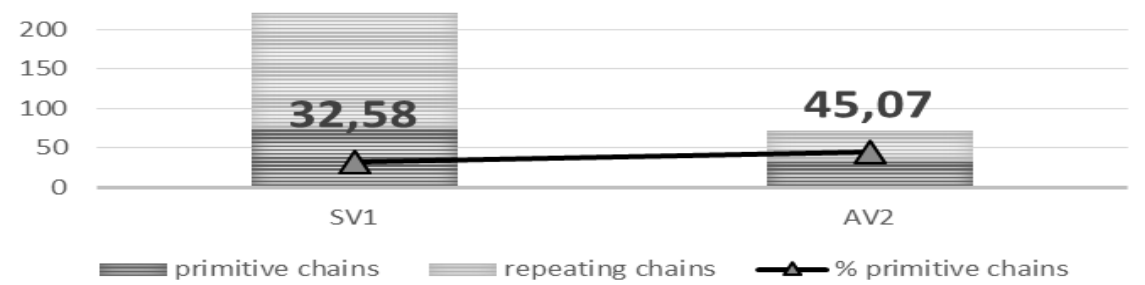

Figure 8. The percentage of primitive chains in both processes (SV1) and (AV2). 


\subsection{Primitive chains position in the process}

In order to understand the deep logic of this creative difference, we compared the cognitive contents of the primitive chains in each process. This showed the existence of fifteen common primitive chains between SV1 and AV2.

The first appearance of primitive chain can be anywhere in the process. Representation of links between the common primitive chains appearances allows the understanding of their positioning logic into both process. Figure 9 visualize the location of the common primitive chains with respect to each process.

The images visualization of precedent changes the location of common primitive chains in the design process and their order of succession.

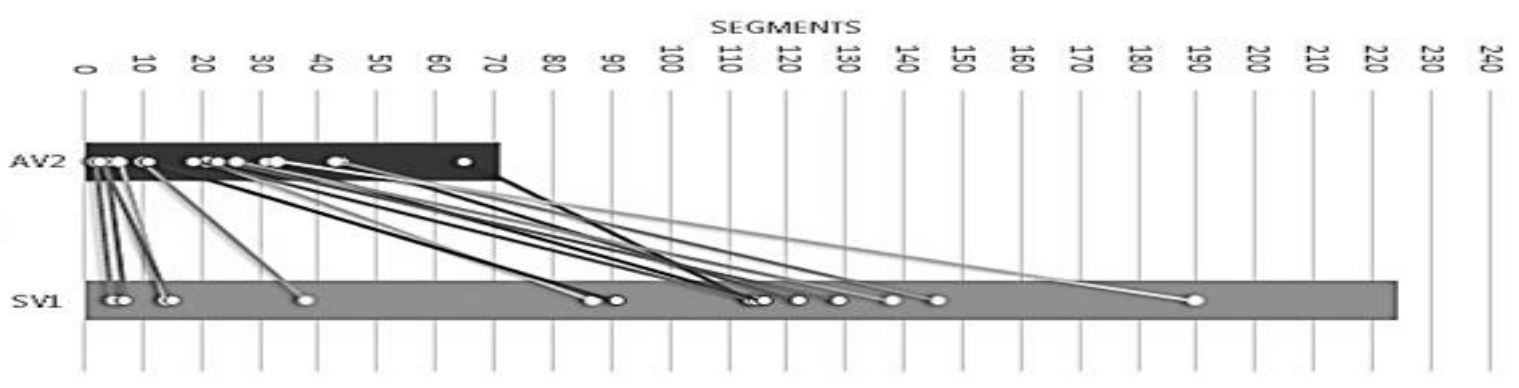

Figure 9. The appearance moment and the link between locations of the common primitive chains in both processes.
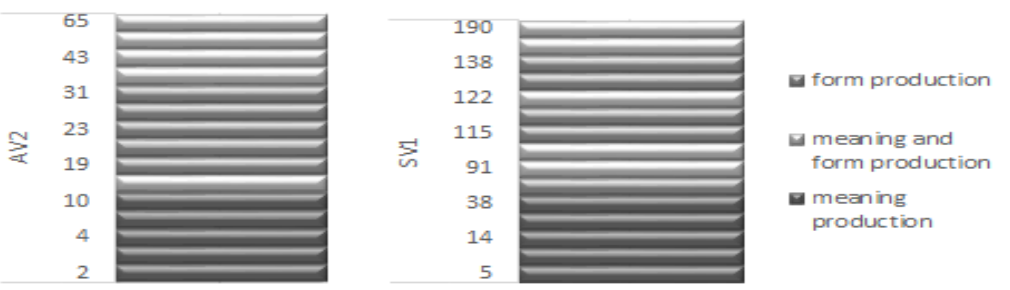

Figure. 10 The cognitive content of common primitive chains of the tow processes, respectively to their appearance order.

The primitive chains of form production and chains of both meaning and form production, which intermingle in SV1, are more orderly in AV2 (Figure 10). Images visualization of precedents organizes and orders the deep structure. It provides a succession of homogeneous primitive chains. This result indicate a homogeneity of the process explained by the existence of a deep order (of the deep structure) that organizes the design activity.

\section{CONCLUSION}

Unlike works that measure the impact of examples visualisation on the design outcome, our study measures the impact of such practice on the designer's cognitive process. We used a protocol analysis to elaborate and study the experimental work of two architects. We applied a semio-morphic coding scheme to identify the active content of the process and its cognitive actions.

Our results indicate that viewing images of precedents' increases the productivity of the architectural idea generation process and that by increasing its efficiency and effectiveness. Efficiency reduces the length of the process and the number of the invested design actions, making thus a more thoughtful process. While effectiveness reduces the repetition of the same cognitive actions.

We equally find that visualization of images affects the morphology of the process by limiting its exploratory aspect enhancing by the way the homogeneity of each of its cognitive stages. The process becomes then more productive, more structured and more organised. It begins with a short and more efficient semic phase that allows an early apparition of the morphic work.

Beyond the improvement of its performances, images visualisation also make the cognitive process of ideation more creative. It allows in fact, a faster, easier and more ordered emergence of primitive chains of actions. 
This research has partially demonstrated how viewing images of examples derived from internet affects the cognitive content of the process. This beneficial effect on the idea generation process indicate that image viewing could be considered as a design aid, to be confirmed in further studies with ample samples.

\section{REFERENCES}

Arrouf, A. (2012), "Vers une théorie scientifique de la conception architecturale. Contribution à l'épistémologie architecturale et à la modélisation de l'acte de concevoir", Editions Universitaires Européennes, Saarinen, Allemagne.

Arrouf, A. and Bensaci, A. (2006), "Modélisation du processus de conception, Etude expérimentale du système compositionnel, instance conception". Courrier du savoir, Vol. 7 No. Décembre 2006, pp. 67-86.

Boudon, P. (1994), "Enseigner la conception architecturale, cours d'architecturologie", Savoir-faire pour l'architecture, Edition de la Villette, Paris.

Cai, H., Do, E.Y.L. and Zimring, C.M. (2010), "Extended linkography and distance graph in design evaluation: an empirical study of the dual effects of inspiration sources in creative design", Design studies, Vol. 31 No. 2, pp. 146-168. https://doi.org/10.1016/j.destud.2009.12.003

Casakin, H. and Goldschmidt, G. (2000), "Reasoning by visual analogy in design problem-solving: the role of guidance". Journal of Planning and Design in Environment \& Planning B, Vol. 27, pp. 105-119. https://doi.org/10.1068/b2565

Dubois, J. (1994), "Lexis, Dictionnaire de la langue française", Larousse, Paris.

Ericsson, K. A. and Simon, H. A. (1980), "Verbal reports as data", Psychological Review, Vol. 87 No. 3 , pp. 215-251. https://doi.org/10.1037//0033-295x.87.3.215

Finke, R. (1990), "Creative imagery: Discoveries and inventions in visualization". Erlbaum, Hillsdale, NJ.

Gero, J. S. and Tang, H-H. (2001), "The differences between retrospective and concurrent protocols in revealing the process-oriented aspects of the design process", Design Studies, Vol. 21 No. 3, pp. 283-295. https://doi.org/10.1016/s0142-694x(00)00030-2

Goldschmidt, G. (1991), “The Dialectics of sketching”, in Creativity Research Journal, Vol. 4, pp. 123-143. https://doi.org/10.1080/10400419109534381

Goldschmidt, G. (1995), "The designer as a team of one”, in Design Studies, Vol. 16 No. 2, pp. 189-209. https://doi.org/10.1016/0142-694x(94)00009-3

Goldschmidt, G. and Dan, T. (2005), "How good are good ideas? Correlates of design creativity", in Design Studies, Vol. 26, pp. 593-611. https://doi.org/10.1016/j.destud.2005.02.004

Goldschmidt, G. and Maria, S. (2006), "Variances in the impact of visual stimuli on design problem solving performance", in Design Studies, Vol. 27, pp. 549-569. https://doi.org/10.1016/j.destud.2006.01.002

Hay, L., Duffy, A.H.B., McTeague, C., Pidgeon, L.M., Vuletic, T. and Grealy, M. (2017), "A systematic review of protocol studies on conceptual design cognition: Design as search and exploration". Design Science, Vol. 3. https://doi.org/10.1017/dsj.2017.11

Herring, S.R., Chang, C.C., Krantzler, J. and Bailey, B.P. (2009), "Getting inspired!: understanding how and why examples are used in creative design practice". In Proceedings of the SIGCHI Conference on Human Factors in Computing Systems, pp. 87-96. ACM. https://doi.org/10.1145/1518701.1518717

Jansson, D.G. and Smith, S.M. (1991), "Design fixation", in Design Studies, Vol. 12 No. 1, pp. 3-11. https://doi.org/10.1016/0142-694x(91)90003-f

Mc. Neill, T., Gero, J. S. and Warren, J. (1998), "Understanding conceptual electronic design using protocol analysis". Research in Engineering Design, Vol. 10, pp. 129-140. https://doi.org/10.1007/bf01607155

Malaga, R.A. (2000), 'Effect of stimulus modes and associative distance in individual creativity support systems', In Decision Support Systems, Vol. 29 No. 2, pp. 125-141. https://doi.org/10.1016/S01679236(00)00067-1.

Perttula, M. and Sipila, P.(2007), "The idea exposure paradigm in design idea generation", in Journal of Engineering Design, Vol. 18 No. 1, pp. 93-102. https://doi.org/10.1080/09544820600679679

Sun, G., Yao, S. and Carretero, J.A. (2014), "Comparing Cognitive Efficiency of Experienced and Inexperienced Designers in Conceptual Design Processes", In Journal of Cognitive Engineering and Decision Making, Vol. 8 No. 4, pp. 330-351. http://doi.org/10.1177/1555343414540172.

Suwa, M., Purcell, T. and Gero, J.S. (1998), "Macroscopic Analysis of Design processes based on a scheme for coding designer's cognitive actions", Design Studies, Vol. 19, pp. 455-483. https://doi.org/10.1016/s0142694x(98)00016-7

Suwa, M. and Tversky, B. (1997), "What do architects and students perceive in their design sketches? A protocol analysis”, in Design Studies, Vol. 18 No. 4, pp. 385-403. https://doi.org/10.1016/s0142-694x(97)00008-2

Tang, H-H. and Gero, J.S. (2002), “A cognitive method to measure potential creativity in designing”. In Workshop 17, Creative systems: Approaches to creativity in AI and cognitive science, ECAI-02, pp. 47-54. 